\title{
Cuckoo Search Optimization for Reduction of a Greenhouse Climate Model
}

\author{
Hasni Abdelhafid \\ Faculty of Technology, University of Bechar \\ BP 417, 08000, Algeria \\ Email:hasni_haf@yahoo.fr
}

\author{
Sehli Abdelkrim \\ Faculty of Technology, University of Bechar \\ BP 417, 08000, Algeria
}

\author{
Haffane Ahmed \\ Faculty of Technology, University of Bechar \\ BP 417, 08000, Algeria
}

\author{
Draoui Belkacem \\ Faculty of Technology, University of Bechar \\ BP 417, 08000, Algeria
}

\begin{abstract}
Greenhouse climate and crop models and specially reduced models are necessary for bettering environmental management and control ability. In this paper, we present a new metaheuristic method, called Cuckoo Search (CS) algorithm, established on the life of a bird family for selecting the parameters of a reduced model which optimizes their choice by minimizing a cost function. The reduced model was already developed for control purposes and published in the literature. The proposed models target at simulating and predicting the greenhouse environment. [?]. This study focuses on the dynamical behaviors of the inside air temperature and pressure using ventilation. Some experimental results are used for model validation, the greenhouse being automated with actuators and sensors connected to a greenhouse control system on the cuckoo search methods to determine the best set of parameters allowing for the convergence of a criteria based on the difference between calculated and observed state variables (inside air temperature and water vapour pressure content). The results shown that the tested Cuckoo Search algorithm allows for a faster convergence towards the optimal solution than classical optimization methods.
\end{abstract}

Keywords-optimization; cuckoo search; greenhouses; metaheuristics; climate models.

\section{INTRODUCTION}

Humanity is going through a crisis which is characterized by a number of problems, the most serious may be mentioned which is that of the highly uneven distribution of agricultural products between different nations. Indeed nearly two-thirds of humanity now living in situation of poverty and deprivation fault of the inadequate use of scientific and technological instruments. In the context of sustainable development, the fight against this serious problem of unequal distribution of agricultural products is part of a social challenge accompanied by a scientific challenge. Indeed many searches have been working for several years studying various technical and scientific means a quantitative improvement of agricultural production. Crops under shelter and specifically greenhouse crops have experienced during the last thirty years a significant expansion. The greenhouses are closed spaces, closed by translucent walls to obtain for agricultural production, better environmental conditions that natural conditions. Thus the greenhouse is a way to transform local outdoor conditions in a more favorable microclimate for plant growth. The technology (heating, cooling, control computers etc ...) allowed the improvement of greenhouses so that they become increasingly sophisticated

The objective of this work is to optimize the mathematical model[?] of the greenhouse to identify some physical parameters of the above model via the Cuckoo Search (CS). This new metaheuristic search algorithm, has been developed by Yang and Deb (2009).

\section{EXPERIMENTAL DATA}

All the experimental data used in this work have been collected between May 14 and 22 1991, in a 416m2 double roof plastic house occupied by a tomato-crop and situated near Avignon in the South of France.

\section{FORMULATION OF THE PROBLEM}

Optimization is a phenomenon of selecting the proper variable from given variables, defining an objective function in a given planning space, and searching for a minimum (or a maximum) value within boundary constraints. The objective of this work is to optimize a reduced greenhouse model which controlled variables are interior temperature and humidity and actuators are the vapor system, the vent opening, the soil and the air heating.

Heat and water vapour balances have been first formulated in order to get the key equations of the complete model. Then exacting equations have been added to complete the model.

TABLE I: Notations

\begin{tabular}{|c|c|}
\hline$Q_{s}$ & Air heating loads $\left(\mathrm{Wm}^{-2}\right)$ \\
\hline$Q_{s}$ & Soil heating loads $\left(\mathrm{Wm}^{-2}\right)$ \\
\hline$\varphi_{l}$ & Injected evaporative cooling by fog system $\left(\mathrm{Wm}^{-2}\right)$ \\
\hline$P T_{i}$ & Water vapor saturation pressure at $T_{i}(\mathrm{hPa})$ \\
\hline$V$ & Wind speed $(\mathrm{m} / \mathrm{s})$ \\
\hline$s$ & Vents opening surface $\left(\mathrm{m}^{2}\right)$ \\
\hline$T_{i}$ & Indoor temperature $\left({ }^{\circ} \mathrm{C}\right)$ \\
\hline$T_{e}$ & outdoor temperature $\left({ }^{\circ} \mathrm{C}\right)$ \\
\hline$P_{i}$ & Indoor pressure $(\mathrm{hPa})$ \\
\hline$P_{e}$ & outdoor pressure $(\mathrm{hPa})$ \\
\hline$R_{g}$ & Outside global radiation $\left(\mathrm{Wm} \mathrm{m}^{-2}\right)$ \\
\hline
\end{tabular}




\section{PROBLEM OF IDENTIFICATION}

As it described with supplementary details in a precedent paper[?], in the case of greenhouse the identification techniques need a system approach of the mass and thermal transfers which can be qualified by four kinds of variables describing the greenhouse and its environment: the entry vector , which describes the initial conditions from which the system evolves; the output vector, or the set of state variables which can be observed and measured; the current state of the system, which includes the state variables of the system evolving as a function of time and the vectors of unknown system parameters. The dynamic behavior of the system can be described by a ensemble of tow equations:

A state equation:

$$
\frac{d X(t)}{d t}=f[X(t), U(t), P]
$$

An observation equation:

$$
Y(t)=g[X(t), U(t), P]
$$

\section{PHYSICAL MODELING OF GREENHOUSE CLIMATE}

\section{A. The heat and water vapour balances}

In order to reduce the system order of the thermal model, an empirical approach based on considerations about the characteristic time scales of each thermal component of the system is considered, two main components are examined:

- The soil and hefty structural elements. With characteristic time scale much longer than the observation time scale. They will be jointly gathered beneath the form of a virtual thermal mass characterized by the virtual temperature $T_{m}$ and thermal capacity $C_{m}$.

- $\quad$ The crop greenhouse superstructure and the inclosed air space, which characteristic time scale $\left(\tau_{c}\right)$ is feeble $\left(200<\tau_{c}<500 s\right)$ and enough like to the observance time ladder (3600sor 900 s), which can be typify by the temperature $T_{i}$ and water vapour pressure $P_{i}$.

The equation (??) represente the the equation of the virtual thermal mass [?] :

$$
C_{m} \frac{d T_{m}}{d t}=h\left(T_{i}-T_{m}\right)+Q_{s}+\beta R_{g}
$$

where the first term on the right hand side is the heat exchanged with the greenhouse air, the second one is the soil heating flux and the last one, the solar gain directly absorbed by the thermal mass.

Ignoring air inertia in front of the hefty structure, one can perform the air thermal balance as follows [?]:

$$
0=\alpha R_{g}+Q_{a}+h\left(T_{m}-T_{i}\right)+K\left(T_{e}-T_{i}\right)+K_{l}\left(P_{e}-P_{i}\right)
$$

where the first term on the right hand side is the solar gain, the second one the air heating, the third one the thermal exchange with the thermal mass, the fourth one is the overall heat exchange between inside and outside and the fifth and last term represents the sensible and latent heat exchanges by ventilation and leakages.

The air water vapour balance takes into consideration the crop transpiration, the water vapour added by fogging and the interchange with exterior, it can be depict by the equation(??) [?]:

$$
C_{l} \frac{d P_{i}}{d t}=A \tau R_{g}+B\left(P T_{i}-P_{i}\right)-K_{l}\left(P_{i}-P_{e}\right)+\varphi_{l}
$$

Whither the first term of the right hand side depicts the crop transpiration (merely described as a linear function of overall radiation and saturation deficit), the second one the interchanges by ventilation and the final one the contribution of the fog system [?].

\section{B. Solving the equations}

Simultaneous integration of the equations of energy ((??) and (??)) and water vapour balances (??) leads to a system of tree equations with tree unknowns $\left(T_{m}, T_{i}, P_{i}\right)$ who may be introduc in a recursive form as a function of the past (time $\mathrm{n})$, the instantaneous input vectors $\left(R_{g}, T_{0}, V, P_{0}, P T_{i}\right)$, of the command variable $\left(Q_{s}, Q_{a}, \varphi_{l}\right)$ and of model parameters ( inclusive in the matrices line) which are partially to be identified. [?]:

The complete system can then be represented is as follows

$$
\begin{gathered}
P_{i(n+1)}=P_{i(n)} \exp (-\xi \Delta t)+(1-\exp (-\xi \Delta t)) \ldots \\
\times\left(\frac{r S B \gamma \tau^{\prime}}{\xi} \frac{\chi}{\xi} \frac{\gamma S B}{\xi} \frac{\gamma S}{\xi}\right) \times\left(R_{g}, P_{e}, P T_{i}, \varphi_{l}\right)^{\prime} \\
\xi=\frac{(A l \sqrt{C} s V)+\left(A l \sqrt{C} s_{0} V\right)+d_{0}+\left(\frac{B \gamma S}{\rho C_{p}}\right)}{v} \\
\xi=\left(\rho C_{p} A l \sqrt{C} s V\right)+\left(\rho C_{p} A l \sqrt{C} s_{0} V\right)+\rho C_{p} d_{0}+(B \gamma S) \\
\chi=\xi-B \gamma S
\end{gathered}
$$

The equation (??) represente the Inside greenhouse temperature[?][?] :

$$
\begin{aligned}
T_{i(n+1)} & =\frac{h}{v} T_{m(n+1)}+\left(\frac{v-h}{v} \frac{\alpha}{v} \frac{1}{v} \frac{K_{l}}{v} \frac{-K_{l}}{v}\right) \ldots \\
& \times\left(T_{e}, R_{g}, Q_{a}, P_{e}, P_{i}\right)^{\prime}
\end{aligned}
$$

where

$$
\begin{aligned}
T_{m(n+1)} & =T_{m(n)} \exp \left(-\frac{\Delta t}{\tau}\right)+\left(1-\exp \left(-\frac{\Delta t}{\tau}\right) \ldots\right. \\
& \times \delta \times\left(T_{e}, R_{g}, Q_{s}, Q_{a}, P_{e}, P_{i}\right)^{\prime}
\end{aligned}
$$

and

$$
\delta=\left(1 \frac{\alpha h+\beta v}{h\left(k+k_{s}\right)} \frac{v}{h\left(k+k_{s}\right)} \frac{1}{\left(k+k_{s}\right)} \frac{K_{l}}{\left(k+k_{s}\right)} \frac{-K_{l}}{\left(k+k_{l}\right)}\right)
$$


Fig. 1: Block Diagram of the Controlled greenhouse.

Fig. 2: Measured air temperature inside the greenhouses between May 14 and 22.

Fig. 3: Measured air water vapour pressure inside the greenhouses between May 14 and 22.

\section{PRINCIPLE OF MODEL PARAMETERS IDENTIFICATION}

\section{THE RESUlts OF THE BLOCK DIAGRAM AND THE OPEN LOOP}

The Fig ?? represent the block diagram of the greenhouse together with the four actuators: $s, Q_{a}, Q_{s}, \varphi_{l}$; five input variables have also been considered : $T_{e}, P_{e}, R_{g}, V, P T_{i}$; which are considered as disturbances in the control loop.

Some simulations have prior been carried out to study the dynamic behavior of the controlled variables (see Fig ??). In these assay, the initial conditions for interior temperature $T_{m(n)}$ and water vapour pressure $P_{i(n)}$ are considered.

Figure ?? and Figure ?? shows the experimontal of temperature and water vapour pressure at the interior of the greenhouses during more then one week.

The elevated number of parameters to be identified leads to fix some of them, especially those which are already known with a good accuracy and particularly:

- $K$ the overall heat loss coefficient through the greenhouse cover $\left(W^{-2} K^{-1}\right)$ is [?]:

$$
K=7.6+0.42 V
$$

- $A l \sqrt{C}$ is a dimensionless parameter of the model of natural ventilation, it is set to: 0,2 following previous air exchange rate studies on this particular greenhouse [?].

- $\quad s_{0}$ the leakage surface $\left(\mathrm{m}^{2}\right)$ set to $s_{0}=0.7 \mathrm{~m}^{2}$ and $d_{0}$ the leakage $\left(\mathrm{m}^{3} \mathrm{~s}^{-1}\right)$ who are separate of wind speed with $d_{0}=0.6 m^{3} s^{-1}[?]$.

The six reminding parameters of the temperature and pressure balance equations to be optimized are the following:

$T_{m 0}$ : The initial thermal masse temperature $\left({ }^{\circ} \mathrm{C}\right)$

$h$ : the air/ sol convective Exchange coefficient $\left(W^{-2} K^{-1}\right)$

$\alpha$ : the rate absorption of the global radiation by the aerial compartment of the greenhouse.

$\tau$ : the time constant or characteristic time $(s)$.

$\beta$ : the rate of absorption of the global radiation by the thermal mass compartment of the greenhouse.

$B$ : a parameter of the model of transpiration $\left(\mathrm{Wm}^{-2} h \mathrm{~Pa}^{-1}\right)$

Table ?? gives the values of these parameters calculated by the classical algorithm [?]. Parameter values together with their confidence intervals were identified utilizing the classical algorithm, yet T.Boulard and B.Draoui proved that the final
TABLE II: Identified values of the parameters $T_{m 0}, h, \alpha, \tau, \beta$ , $B$ during a one week sequence using the classical algorithm.

\begin{tabular}{|c|c|c|c|c|c|}
\hline$T_{m 0}$ & $h$ & $\alpha$ & $\tau$ & $\beta$ & $B$ \\
\hline 16 & 13.5 & 0.53 & 1058 & 0 & 3.78 \\
\hline
\end{tabular}

TABLE III: Search space of the parameters to be identified.

\begin{tabular}{|c|c|c|c|c|c|}
\hline$T_{m 0}$ & $h$ & $\alpha$ & $\tau$ & $\beta$ & $B$ \\
\hline 14 & 0 & 0.2 & 2 & 0 & 1 \\
\hline 28 & 30 & 0.8 & 1100 & 0.2 & 9.5 \\
\hline
\end{tabular}

result was strongly dependent on the initial values of every parameter. The existence of very large interactions between variables was also shown and especially it's can be seen that one part of the variability of the results which is due to one model parameter can be attributed to another one if the two model parameters are statistically strongly correlated [?].

\section{SEARCH SPACE OF PARAMETERS}

With the classical Algorithm, the parameters to be optimized are selected, one must define also their numerical bound. For exemplar, the temperature of the thermal mass varies between $14.2^{\circ}$ and $29.7^{\circ}$ (average minimal and maximal temperatures observed in Avignon, France during May.). The search space for each parameters are given in Table ??, Search space of the parameters $T_{m 0}, h, \alpha, \tau, \beta, B$ to be identified.

\section{OBJECTIVE FUNCTIONS:}

As objective function, the equations of air temperature and pressure $\left(T_{i}\right.$ and $\left.P_{i}\right)$ are considered and defined by the relations (??) and (??), the objective of this study is to minimize the difference between measured and calculated values with the selected parameters.

\section{Cuckoo SEARCH}

\section{A. Cuckoo Breeding Behaviour.}

CS is based on the reproduction strategy of some cuckoos species augmented by a Levy flight behaviour found in the foraging habits of other animal species.

Cuckoos are nest parasite, they lay their eggs in other birds nests and leave the host birds to incubate and rear their young.

When the Cuckoo nestling hatches, it instinctively pushes the other eggs and nestlings out of the nest. This reproductive strategy can be extremely costly for foster parents, because the reproductive success of parasitized hosts is dramatically reduced, and in most cases (depending on parasite and host species) is nil [?]; which, Leads to strong host adaptations to detect and reject foreign eggs or to simply abandon their nests and build new ones. As a result of these Female cuckoos developed counter-adaptations: host-egg mimesis, they occasionally specializes in using a private host species, and lay eggs that closely resemble the host eggs. This, Coevolutionary dynamics donate rise to an evolutionary antagonistic arms race between the two species With growing fitness costs of parasitism, choice for host defences increases, which in turn may force parasites to specialize and evolve fine-tuned adaptations that overcome a particular host's defences. [?]. 


\section{B. Lvy Flights}

The issue is how animals get nourishment in dynamic natural milieu where they have sparsely or no acquaintance of where resources are situated. Foraging theory foretell that Lvy steal move optimize the hit of resources random searches. [?] In recent years, biologists have uncovered that Lvy flights describe foraging patterns in a number of kind of animals and insects: ants, bee.... [?] , in the foraging of marine predators [?] end even in foraging movement patterns of human huntergatherers [?] Lvy flights, appointed by the French mathematician Paul Lvy, are a peculiar class of random walks. A random walk is a formalization of the intuitive idea of taking successive steps, each in a random direction.Thus, they are easy stochastic processes consisting of a discreet sequence of shifting events (i.e. move lengths) separated by successive reorientation events (i.e. turning angles). [?] The statistical dispensation of shifting lengths and changes of direction, depict the stochastic process. In particular, Lvy random walk models imply a uniform dispensation for the turning angles and a Lvy-stable dispensation for the move or flight step length. [?]

In Lvy flights, the lengths, $l$, of the steps or jumps of the walks are distributed as a power law [?],

$$
P(l)=l^{-\lambda},(1<\lambda \leq 3)
$$

Lvy flights, are typify by the being of scarce but exceedingly large steps, alternating between sequences of many short-length leaps and the same sites are revisited much fewer frequently than in patterns described by other process.

\section{Cuckoo Search algorithm}

CS is a population-based algorithm, in a way like to genetic algorithm. Where the solution are represented by eggs in hosts nests and the cuckoo eggs represent the further solutions, the goal is to use the new and potentially better solutions (cuckoos) to substitute the bad solutions in the nests.[?] rules:

The CS can be described using following three idealized

1) Each cuckoo lays one egg at a time, and dumps it in a randomly chosen nest;

2) The best nests with high quality of eggs (solutions) will carry over to the next generations.

3) The number of available host nests is fixed, and a host can discover an alien egg with probability $P_{a} \in[0,1]$. In this case, the host bird can either throw the egg away or abandon the nest to build a completely new nest in a new location[?].

The third assumptions can be approximated as the fraction $P_{a}$ of the $n$ nests is replaced by new nests (new random solutions).

The quality or fitness of a solution can be defined in a similar way to the fitness function in genetic algorithms.

The basic steps of C.S are described in the following pseudo code [?]:

\section{Begin}

Objective function $f(X), X=\left(x_{1}, x_{2}, \ldots, x_{d}\right)$;

Generate initial population of $n$ host nests $X_{i},(i=1,2, n)$ while ( $\mathrm{t}<$ MaxGeneration) or (stop criterion) do

Get a cuckoo randomly by Levy flights evaluate its quality/fitness $F_{i}$

Choose a nest among $\mathrm{n}($ say, $\mathrm{j})$ randomly

if $\left(F_{i}>F_{j}\right)$ then

replace $\mathrm{j}$ by the new solution;

\section{end if}

A fraction (pa) of worse nests

are abandoned and new ones are built;

Keep the best solutions

(or nests with quality solutions);

Rank the solutions and find the current best

\section{end while}

Postprocess results and visualization

End

When generating new solutions $x^{(t+1)}$ for a cuckoo $\mathrm{i}$, a Lèvy flight is performed using the following equation:

$$
x_{i}^{t+1}=x_{i}^{t}+\alpha \bigotimes \operatorname{Levy}(\lambda)
$$

where $\alpha>0$ represents a step size. This step size should be related to the scales of problem the algorithm is trying to solve.

The product $\otimes$ means entry-wise multiplications. Lèvy flights essentially provide a random walk while their random steps are drawn from a Levy distribution for large steps.

$$
\text { Levy } \sim u=t^{-\lambda},(1<\lambda \leq 3)
$$

It is worth pointing out that, in the real world, if a cuckoos egg is very similar to a hosts eggs, then this cuckoos egg is less likely to be discovered, thus the fitness should be related to the difference in solutions. Therefore, it is a good idea to do a random walk in a biased way with some random step sizes.

From the implementation point of view, the generation of random numbers with Lèvy flights consists of two steps: the choice of a random direction and the generation of steps which obey the chosen Lèvy distribution. The generation of a direction should be drawn from a uniform distribution, while for the generation of steps there are a few ways, but one of the most efficient and yet straightforward ways is to use the so-called Mantegna algorithm for a symmetric Lèvy stable distribution. Here symmetric means that the steps can be positive and negative.[?] by

In Mantegnas algorithm, the step length s can be calculated

$$
S=\frac{u}{|y|^{1 / \beta}}
$$

Where $0<\beta \leq 2$ is an index. $\mathrm{u}$ and $\mathrm{v}$ are stochastic variables drawn from normal distributions. That is[?]:

$$
\begin{gathered}
u \sim N\left(0, \sigma_{u}^{2}\right), v \sim N\left(0, \sigma_{v}^{2}\right. \\
\sigma_{u}=\left\{\frac{\Gamma(1+\beta) \sin (\pi \beta / 2)}{\left.\Gamma[(1+\beta) / 2] \beta \cdot 2^{(\beta-1) / 2}\right\}^{1 / \beta}}\right.
\end{gathered}
$$

Here $\Gamma(z)$ is the Gamma function,

$$
\Gamma(x)=\int_{0}^{\infty} t^{z-1} e^{-t} d t
$$


TABLE IV: Best parameters result identified by the Cuckoo Search Algorithm.

\begin{tabular}{|c|c|c|c|c|c|}
\hline$T_{m 0}$ & $h$ & $\alpha$ & $\tau$ & $\beta$ & $B$ \\
\hline 23.506 & 3.8093 & 0.65352 & 21.098 & 0.001482 & 8.109 \\
\hline
\end{tabular}

TABLE V: Quadratic errors of air temperature and pressure between model and experimental results according to the identification process .

\begin{tabular}{|c|c|c|}
\hline quadratic error & Classical Model & CS Model \\
\hline Air temperature & 0.1376 & 0.0852 \\
\hline Air pressure & 0.0683 & 0.0415 \\
\hline
\end{tabular}

Fig. 4: Comparaison of the temperature results

Fig. 5: Comparaison of the air water vapour pressure results

\section{RESULTS}

An experimental study of greenhouse was made in a period between 14 and 22 May 1991 situate nearby Avignon in SouthEast of France, this study allowed us to make an identification of a temperature and humidity simulation models. The greenhouse had a tomato-crop area of $416 \mathrm{~m}^{2}$, in a double roof plastic house. Various sensors and actuators correspondant to those which are offer in the Block Diagram of ?? were established and attached to a data logger and control system based on a personal computer and a control card using a sampling interval of 1 hour. The ensemble of parameters given by the Cuckoo Search (CS) algorithm which minimizes the gap between the calculated and experimental output values of the model for a 9 days interlude on an hourly basis is presented in Table ??. Just little seconds are requisite to identify the parameters of the reduced model. In the optimization process, the fraction probability, $\mathrm{Pa}$ (discovery rate) is 0.25 and the Maximum iterations is 100 and the number of nest (size of population) is 50 .

The comparison of the results given by the models optimized with the Cuckoo Search Algorithm or the classical Algorithm with respect to the experimental measurements is given by Figure ?? and figure ?? gives.

The figures exemplify the nice accordance which was spotted between the observed results and the simulation found from the Cuckoo Search Algorithm, both in terms of dynamics and intensity of the signal, especially for the air water vapour pressure calculation.

In order to quantize more exactly the ratification of CS algorithm, the quadratic error are calculated between the observed and simulated results for respectively the data issued from the identified model using the Cuckoo Search Algorithm or the classical Algorithm (Table ??), it's can be seen that, the Cuckoo Search Algorithm enhance very significantly the accuracy of the simplified greenhouse model.

\section{CONCLUSION}

In this paper, the Cuckoo Search (CS) method to solve the greenhouse model parameters identification problem is investigated, the determination of the physical parameters relating the interactions between crop and climate in a horticultural greenhouse can be considerably enhanced both in terms of calculation time and accuracy of the results, via a Cuckoo Search algorithm.

This result is mainly motivating for the reason that this type of biophysical model involves the majority of the time a big number of mechanisms which are not forever exactly modeled or which modeling depends on the particular perspective of the system, such as mainly the biological one. In this case the stage of identification of the model parameters is mainly essential for selecting parameters which are both accurate and robust.

The results showed that the Cuckoo Search algorithm solution quality is better than that of classical Algorithm in most of the test cases. Furthermore, the Cuckoo Search algorithm runs quicker as compared with classical Algorithm.

The advantages of the cuckoo search comprise a uncomplicated organization, instantly accessible for useful applications, easy of realization, speed to obtain solutions and robustness.

For the future research work focuses on exploring another of bettering rendering, while trying to minimize costs, for the reason that this is a means reason for the development productivity.

\section{REFERENCES}

[1] T. Boulard and B. Draoui. In-situ Calibration of a greenhouse climate control model including sensible heat, water vapour and $\mathrm{CO} 2$ balances. IMACS/IFAC bruxelles, BELGIUM; 1995/05/09-12.pVI.A.1-1 ; VI.A.16

[2] T. Boulard and B. Draoui. Natural ventilation of greenhouse with continuous roof vents: Measurements and data analysis. Journal of Agricultural Engineering Research, (61):2736, (1995).

[3] T. Boulard, B. Draoui and F. Neirac, Calibration and validation of a greenhouse climate control model. Workshop: Mathematical and Control Application in Agriculture and Horticulture. Silsoe Grande Bretagne. Acta Horticulturae. 1994

[4] T. Boulard and R. Jemaa. Greenhouse tomato crop transpiration model application to irrigation control. Acta Horticulturae 335. 1993. p 381387.

[5] A. Hasni, and al, Evolutionary Algorithms In The Optimization Of Greenhouse,2008, Climate Model Parameters. International Review on Computers and Software, (I.RE.CO.S.)

[6] J. J Soler and al, Change in host rejection behavior mediated by the preclatory behavior of its brood parasite, Behavioral Ecology, Vol. 10 No. 3: 275-280, (1999).

[7] O Krüger, Brood parasitism selects for no defence in a cuckoo host, Proc. R. Soc. B published online 2 February 2011.

[8] Humphries and al, Foraging success of biological Lvy flights recorded in situ, PNAS, Vol. 109, No. 19: 71697174, (2012).

[9] C. T Brown, Lèvy Flights in Dobe Ju/hoansi Foraging Patterns, Hum Ecol No. 35:129138, (2007).

[10] G. M Viswanathan, Fish in Levy-flight foraging, NATURE, Vol. 465: 1018-1019, ( 2010).

[11] F. Bartumeus, Lèvy processes in animal movement: An evolutionary hypothesis, Fractals, Vol. 15, No. 2: 112, (2007).

[12] Yang XS, Deb S, Cuckoo search via Lèvy fights, Proceeings of World Congress on Nature and Biologically Inspired Computing, India, 210214, (2009). 
[13] Yang XS, Nature-Inspired Metaheuristic Algorithms, Second Edition, Luniver Press, (2010).

[14] B Draoui, Caractrisation et analyse du comportement thermo-hydrique d'une serre horticole. Thse de Doctorat de l'universit de Nice-Sophia Antipolis,(1994).

[15] Arindam Majumder and Dipak Laha, A New Cuckoo Search Algorithm for 2-Machine Robotic Cell Scheduling Problem with Sequence-Dependent Setup Times, Swarm and Evolutionary Computation http://dx.doi.org/10.1016/j.swevo.2016.02.001.

[16] E I Mbuyamba, Active contours driven by Cuckoo Search strategy for brain tumour images segmentation, Expert Systems With Applications 2016 Published by Elsevier Ltd.

[17] Li Huang, Shuai Ding, Shouhao Yu, Juan Wang, Ke Lu, Chaosenhanced Cuckoo search optimization algorithms for global optimization, Applied Mathematical Modelling (2015), 